\title{
Suspension-like hardening behavior of HDPE and time-hardening superposition
}

\author{
Peter C. Roozemond • Vincent Janssens • \\ Peter Van Puyvelde • Gerrit W. M. Peters
}

Received: 8 February 2011 / Revised: 2 May 2011 / Accepted: 6 June 2011 / Published online: 12 July 2011

(C) The Author(s) 2011. This article is published with open access at Springerlink.com

\begin{abstract}
The rheology of solidifying high-density polyethylene (HDPE) is investigated. Experiments on an HDPE were performed with a novel RheoDSC device. Results agree quantitatively with simulations for a suspension of elastic spheres in a viscoelastic matrix except for very low values of space filling $(<5 \%)$, indicating that the rheological behavior of the crystallizing melt in the frequency range investigated is purely suspension like. The hardening behavior of the material is characterized in two different ways; a normalized rheological function and a time-hardening superposition (THS) master curve of rheological properties. An improvement is proposed to the procedure for performing THS that was previously used in the literature. Based on this procedure, a novel method for predicting the rheological properties of crystallizing melts is presented.
\end{abstract}

Keywords Polymer crystallization • Polyethylene • Rheology $\cdot$ Suspension models

\section{Introduction}

Detailed knowledge of the change in rheology of semicrystalline polymers during solidification is of vital im-

P. C. Roozemond · G. W. M. Peters ( $\square)$

Department of Mechanical Engineering,

Eindhoven University of Technology (TU/e),

5600 MB Eindhoven, The Netherlands

e-mail: g.w.m.peters@tue.nl

\section{Janssens · P. Van Puyvelde}

Department of Chemical Engineering, Applied Rheology and Polymer Processing, Leuven Material Research Centre, Katholieke Universiteit Leuven, Leuven, Belgium portance for the modeling of processes such as injection molding, fiber spinning, electrospinning, etc. because the rheological properties, flow field, and crystallization kinetics are all intimately coupled (Custódio et al. 2009; Steenbakkers and Peters 2010). A vast amount of experiments investigating the change of rheological properties can be found in the literature, cf. Acierno and Grizzuti (2003), Boutahar et al. (1998), Han and Wang (1997), Lamberti et al. (2007), Pantani et al. (2001), Pogodina and Winter (1998), and Titomanlio et al. (1997). To quantify the hardening behavior of the material, a normalized rheological function (NRF) is commonly used.

$\Gamma(\xi)=\frac{\left|\eta^{*}(\xi)\right|}{\left|\eta^{*}(\xi=0)\right|}$

where $\Gamma$ is the hardening function, $\xi$ space filling or relative crystallinity, and $\eta^{*}$ the complex viscosity. Among the experimental data gathered, little agreement is found between studies, as shown by Lamberti et al. (2007). This is largely because the experimental procedure commonly used to obtain the hardening curve is prone to experimental errors: rheological properties and space filling (both as a function of time) are separately measured in a rheometer (usually with less precise temperature control) and a differential scanning calorimetry (DSC) apparatus, respectively. Timeviscosity and time-space filling data are then combined to obtain a hardening curve. A slight difference in behavior in the two experiments might result in a large error in the hardening function. For instance, Steenbakkers and Peters (2008) found the experiments from Boutahar et al. (1996, 1998) showed suspension-like behavior but in order to get quantitative agreement 
between experiments and simulations, the space filling obtained from DSC measurements had to be adjusted significantly.

An improvement on this cumbersome method was proposed by Lamberti et al. (2007). They developed a technique that eliminates the need for obtaining space filling and rheological properties at exactly the same temperature. However, experiments were still carried out in two different devices. More recently, the group in Leuven developed a novel apparatus that combines DSC and rheometry: the RheoDSC device (Janssens et al. 2009, 2010; Kiewiet et al. 2008). With this device, rheological and thermal signals can be obtained simultaneously on the same sample and hence both types of experiments are conducted under identical conditions.

Lamberti et al. (2007) studied a novel method to describe the viscosity increase in solidifying polymers. They found that, similar to a time-temperature master curve in time-temperature superposition (TTS), a timehardening master curve can be constructed. An empirical relation for the space filling shift factor was proposed. Custódio et al. (2009) implemented this method of predicting viscosity as a function of space filling in their code for the simulation of shear flow in a multipass rheometer.

A large number of analytical models for the hardening function of suspensions have been developed (Ball and Richmond 1980; Batchelor 1977; Einstein 1906, 1911; Frankel and Acrivos 1967; Graham 1981; Katoaka et al. 1978; Kitano et al. 1981; Krieger and Dougherty 1959; Mooney 1951). However, many of these models are not applicable to crystallizing polymer melts. Empirical models can also be found in abundance in literature (Boutahar et al. 1998; Doufas et al. 2000; Han and Wang 1997; Hieber 2002; Katayama and Yoon 1985; Khanna 1993; Shimizu et al. 1985; Tanner 2003; Titomanlio et al. 1997; Yarin 1992; Ziabicki 1988; Zuidema et al. 2001), but there is little agreement between these models. Thus, there is need for a model that is able to accurately describe rheological properties of semi-crystalline polymers during solidification.

Steenbakkers and Peters (2008) reviewed a number of suspension-based models and found that the generalized self-consistent method (GSCM) developed by Christensen (1990); Christensen and Lo (1979, 1986) describes accurately the rheology of crystallizing isotactic polypropylene (iPP). However, the GSCM is not applicable to the low frequency range, where gellike behavior instead of suspension-like behavior is observed (Pogodina and Winter 1998; Pogodina et al. 1999a, b; Winter and Mours 1997). An extension of the GSCM, including percolation (Alberola and Mele 1996), did not solve this problem.
The goal of this study is to investigate the applicability of the generalized self-consistent method to predicting the hardening curve and space filling shift factors of a material. To this end, data from experiments in the novel RheoDSC device on high-density polyethylene (HDPE) will be used for validation purposes. The experimental results and time-hardening superposition of these results are presented in the "Experiments" section. The "Simulations" section shows the comparison between simulations with the GSCM and the experimental results.

\section{Experiments}

A crystallizing HDPE was analyzed in the prototype RheoDSC device. This instrument was constructed starting from two stand-alone commercial instruments: a Q2000 Tzero ${ }^{\mathrm{TM}}$ DSC (TA Instruments) and an ARG2 rheometer (TA Instruments). The DSC apparatus uses the Tzero ${ }^{\mathrm{TM}}$ technology, which is important for the correction of the thermal resistances and capacitances of the various heat flow paths in the cell caused by the modification of the calorimetric cell environment. The AR-G2 rheometer is a sensitive instrument opening the range of ultra-low torques, which is of importance as the diameter of the measurement geometry is chosen to be of comparable dimension as the DSC sensor $(5 \mathrm{~mm})$. For more details the reader is referred to Kiewiet et al. (2008). Both the rheology and thermal signals of the RheoDSC device have been validated, as reported by Janssens et al. $(2009,2010)$ and Kiewiet et al. (2008).

Experiments were performed at $124^{\circ} \mathrm{C}$ at five oscillatory frequencies. Results for the complex viscosity, modulus and phase angle are presented in Fig. 1. Different symbols indicate the increasing space filling. The experiments show a large difference in rheology between the experiments with zero and just six percent relative crystallinity, much larger than what would be caused by a suspension of spherulites with this amount of space filling. The effect is accounted for by extrapolating the data for the crystallizing melt to obtain adjusted values for the rheology of the melt, as will be explained in the "Early stage of crystallization" section. The modulus for the fully crystallized material $(\xi=1)$ is also obtained by extrapolation. Values are shown in Table 1.

\section{Hardening function}

Figure 2 shows the hardening function, calculated using a NRF based on the magnitude of the complex viscosity 


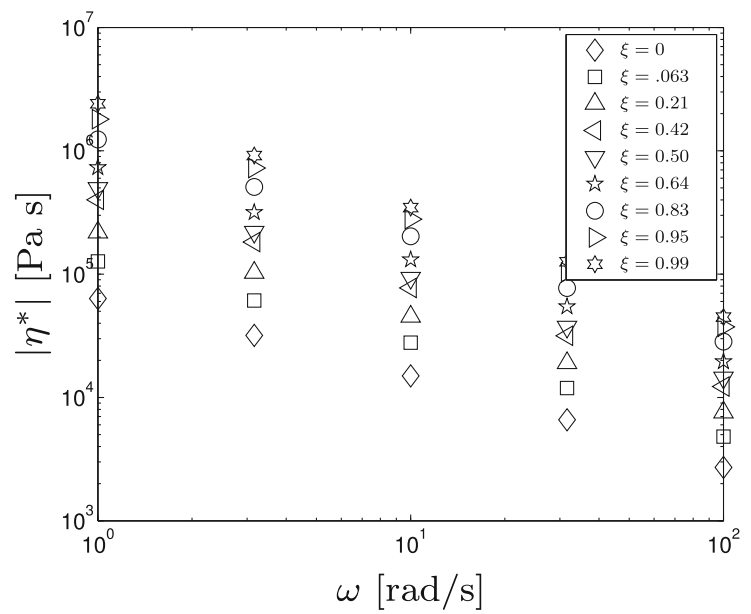

(a)

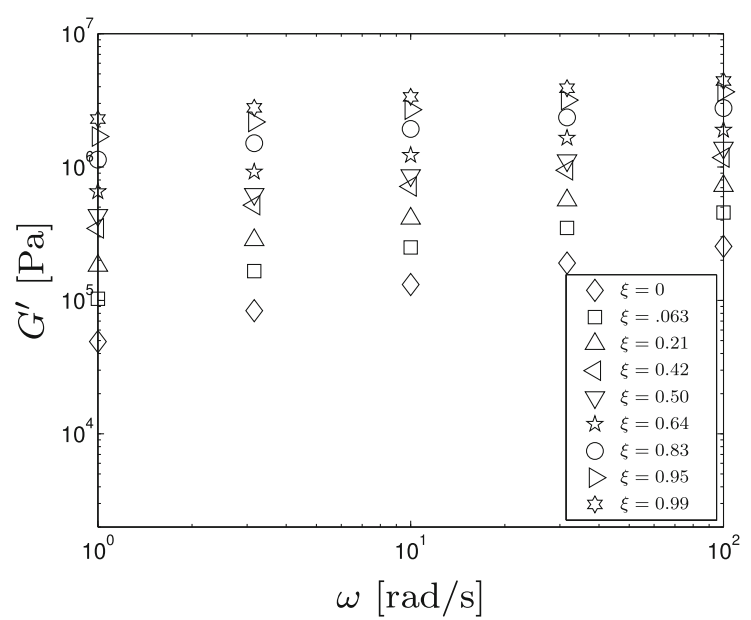

(c)

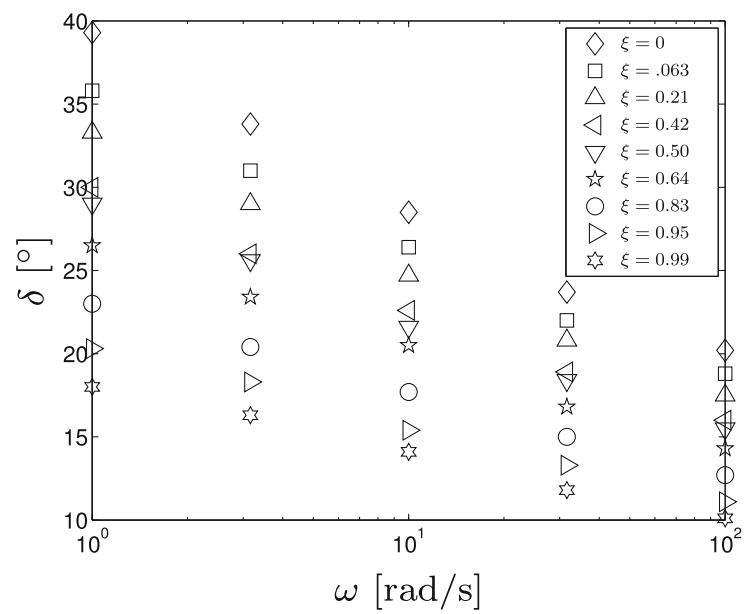

(b)

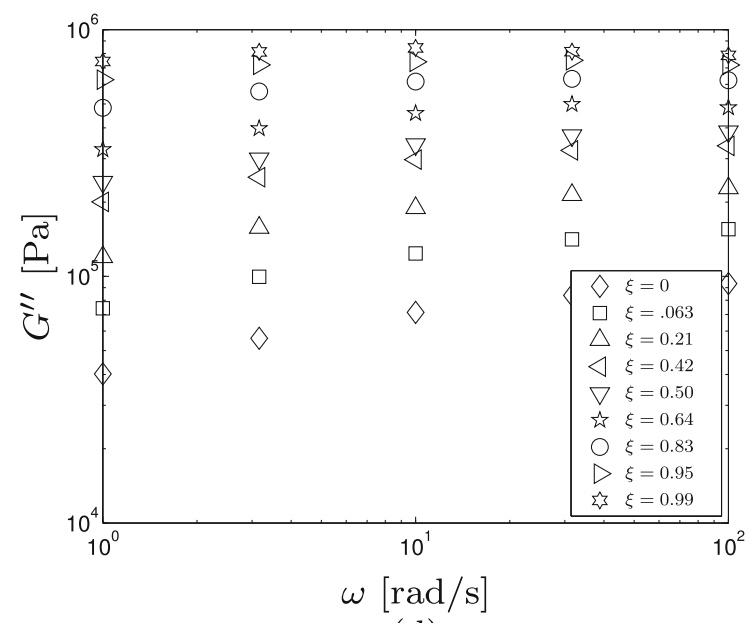

(d)

Fig. 1 Measurements of the magnitude of the complex viscosity (a), phase angle (b), storage modulus (c), and loss modulus (d)

versus space filling for the five frequencies investigated.

The NRF is given by

$\Gamma(\xi, \omega)=\frac{\left|\eta^{*}(\xi, \omega)\right|}{\left|\eta^{*}(\xi=0, \omega)\right|}$.

The hardening curve is similar in shape to what was observed in the literature (Janssens et al. 2010; Lamberti et al. 2007), taking larger values for lower frequencies throughout the range of space filling. It is

Table 1 Storage and loss moduli for the melt, $G_{0}^{*}$, and for the fully crystallized material, $G_{1}^{*}$

\begin{tabular}{|c|c|c|c|c|}
\hline $\begin{array}{l}\omega \\
{[\mathrm{rad} / \mathrm{s}]}\end{array}$ & $\begin{array}{l}G_{0}^{\prime} \\
{[\mathrm{MPa}]}\end{array}$ & $\begin{array}{l}G_{1}^{\prime} \\
{[\mathrm{MPa}]}\end{array}$ & $\begin{array}{l}G_{0}^{\prime \prime} \\
{[\mathrm{MPa}]}\end{array}$ & $\begin{array}{l}G_{1}^{\prime \prime} \\
{[\mathrm{MPa}]}\end{array}$ \\
\hline 1.00 & 0.084 & 2.29 & 0.062 & 0.692 \\
\hline 3.16 & 0.142 & 2.78 & 0.087 & 0.757 \\
\hline 10.0 & 0.223 & 3.36 & 0.121 & 0.786 \\
\hline 31.6 & 0.324 & 3.90 & 0.132 & 0.760 \\
\hline 100 & 0.432 & 4.42 & 0.148 & 0.734 \\
\hline
\end{tabular}

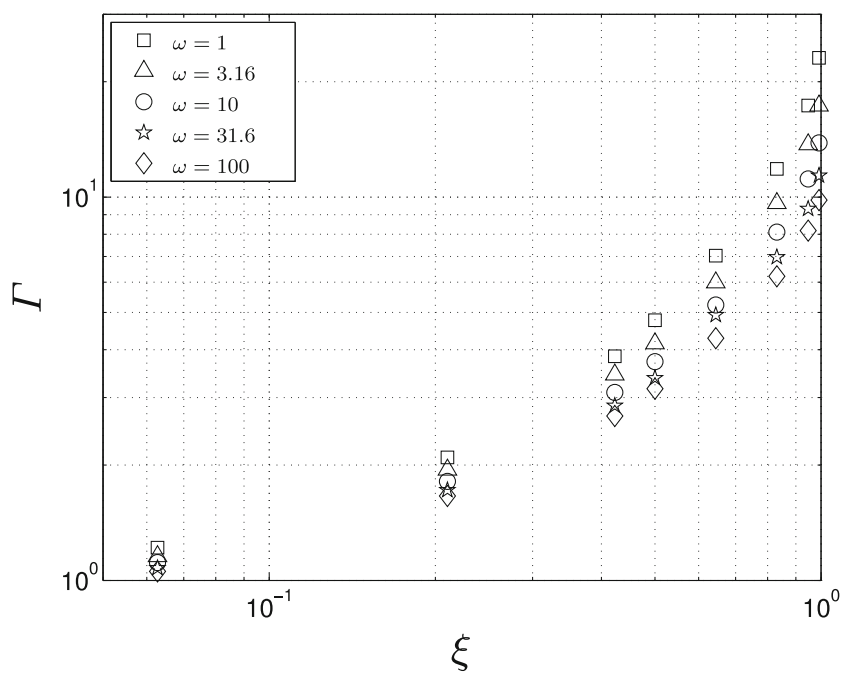

Fig. 2 Hardening curves calculated using Eq. 2 for five frequencies 


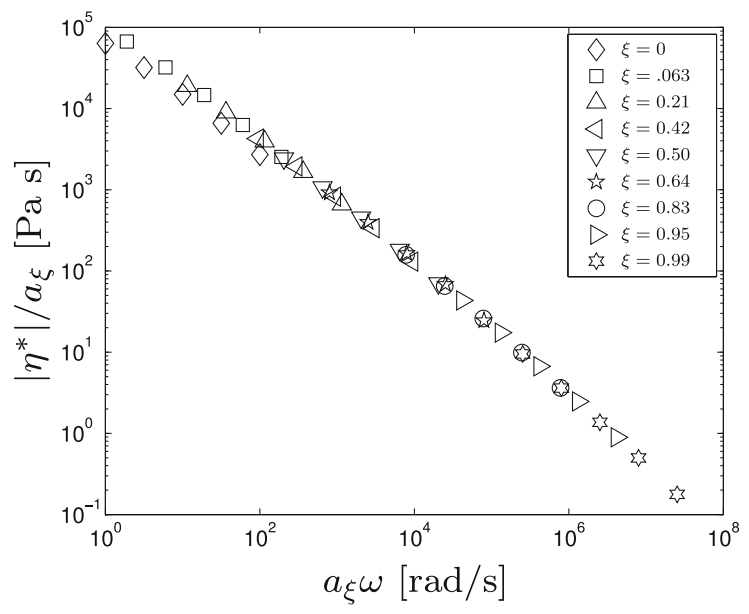

(a)

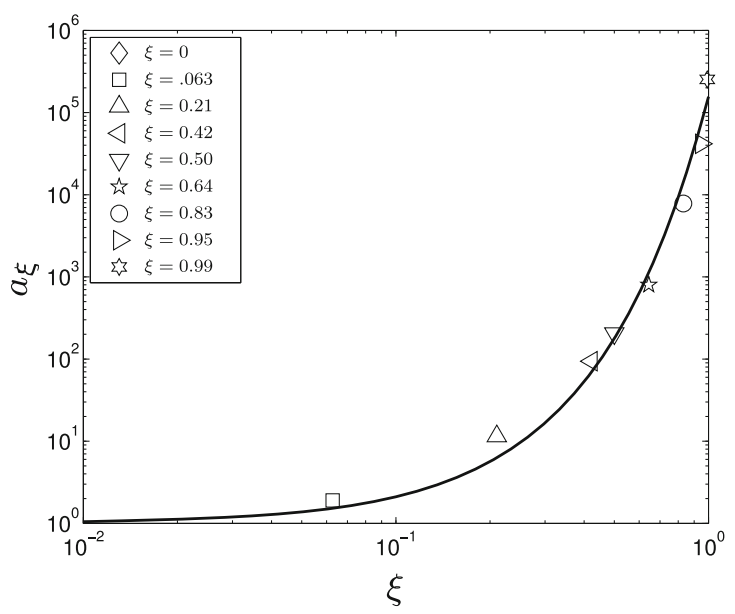

(b)

Fig. 3 Master curve of $\left|\eta^{*}\right|$ shifted on the viscosity (a) and corresponding shift factors (b). The solid line shows a fit with the empirical relation from Lamberti et al. with parameters $\alpha_{1}=5.19$ and $\alpha_{2}=1.20$

emphasized that in accordance with Lamberti et al. (2007) and Janssens et al. (2010), little change in rheology is observed for space filling below $10 \%$, whereas other authors have reported order of magnitude changes for $\xi<10 \%$ (Acierno and Grizzuti 2003; Titomanlio et al. 1997).

Time-hardening superposition

Lamberti et al. (2007) found that in a frequencyviscosity plot, measurements for crystallizing iPP with different space fillings can be shifted to a single master curve, analogous to a TTS master curve. This was done by shifting the relaxation times of the material with $a_{\xi}$, so that the shifted frequency and viscosity become

$$
\begin{aligned}
& \omega_{\text {master }}=\omega * a_{\xi}(\xi) \\
& \eta_{\text {master }}=\eta / a_{\xi}(\xi)
\end{aligned}
$$

Lamberti et al. also found that the shift factor $a_{\xi}$ was reasonably well described by the empirical relation

$\log _{10} a_{\xi}=\alpha_{1} \xi^{\alpha_{2}}$

where $\alpha_{1}$ and $\alpha_{2}$ are fitting parameters.

Viscosity data from the present experiments were shifted in the same way. The resulting master curve is shown in Fig. 3a. The curve for $\xi=0$ corresponds to the values given in Table 1. Shift factor versus space filling is shown in Fig. 3b. The empirical relation from Lamberti et al. is depicted with the solid line. We observe that Lamberti's empirical relation is able to capture our data quite well with parameters $\alpha_{1}=5.19$, $\alpha_{2}=1.20$. Lamberti studied two grades of iPP and found $\alpha_{1}=3.875, \alpha_{2}=1.236$ for one of the grades and $\alpha_{1}=12.073, \alpha_{2}=3.166$ for the other.

Actually, the procedure that Lamberti et al. used to obtain a master curve differs from the way in which TTS is conventionally conducted. In TTS the shift factor for the relaxation times of the material $a_{\xi}$ is obtained by shifting the phase angle and subsequently the master curve for the viscosity would be obtained by applying the shift factor for relaxation times, and an additional vertical shift factor $b_{\xi}$;

$\eta_{\text {master }}=b_{\xi}(\xi) * \eta / a_{\xi}(\xi)$

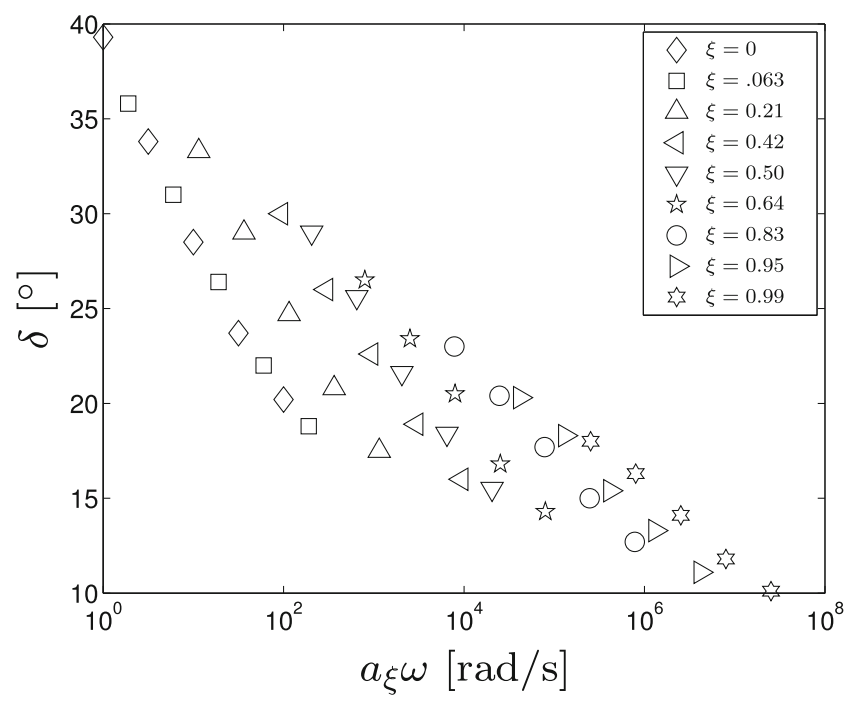

Fig. 4 Master curve for the measured phase angle with shift factors obtained from the one-step shifting procedure 


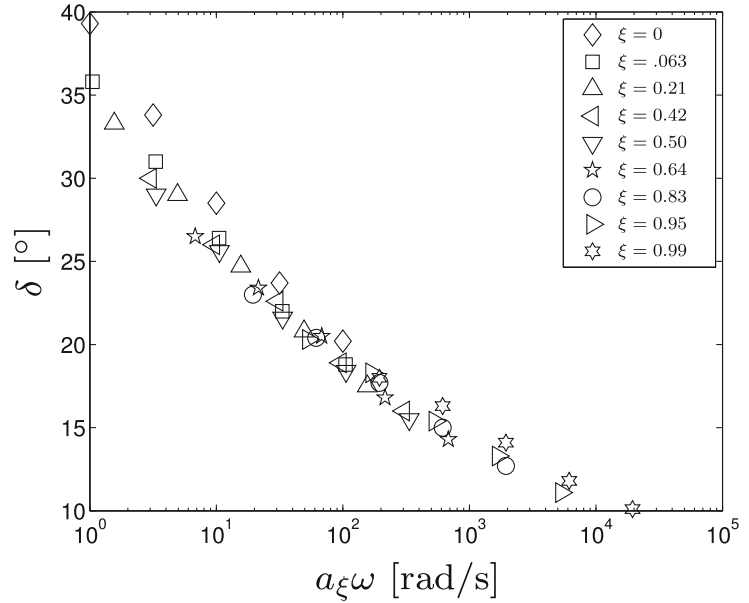

(a)

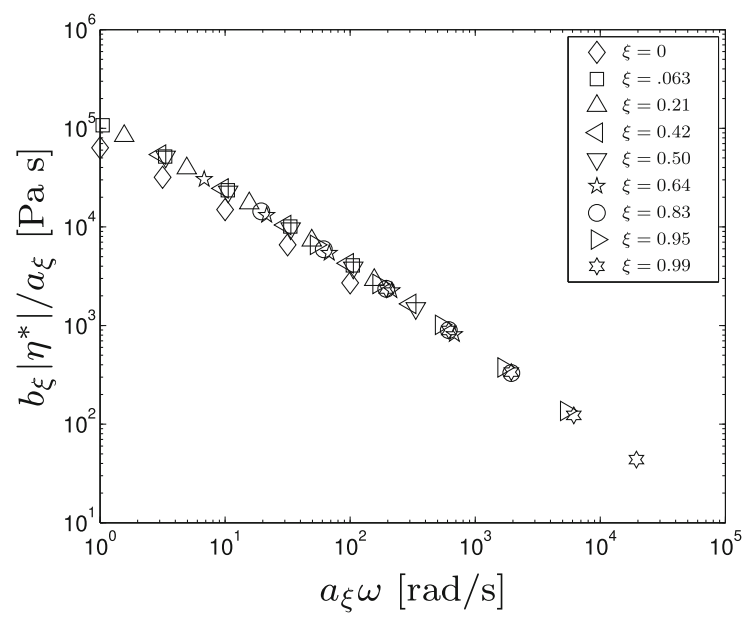

(b)

Fig. 5 Master curves for the measurements of the phase angle (a) and magnitude of the complex viscosity (b). Data shifted using the two-step procedure

If the vertical shift factor $b_{\xi}$ is of order 1 , which is usually the case in TTS, the difference between the one-step procedure that Lamberti et al. used and the conventional two-step shifting procedure is small. However, if we apply Lamberti's procedure, i.e., obtaining $a_{\xi}$ through shifting the complex viscosity, to our measurements, we observe that the phase angle curves shifted with this $a_{\xi}$ do not form a single master curve. This is shown in Fig. 4. This means that although this procedure could be used to calculate viscosity as a function of space filling, $a_{\xi}$ does not shift the relaxation times of the material. Therefore, the viscosity master

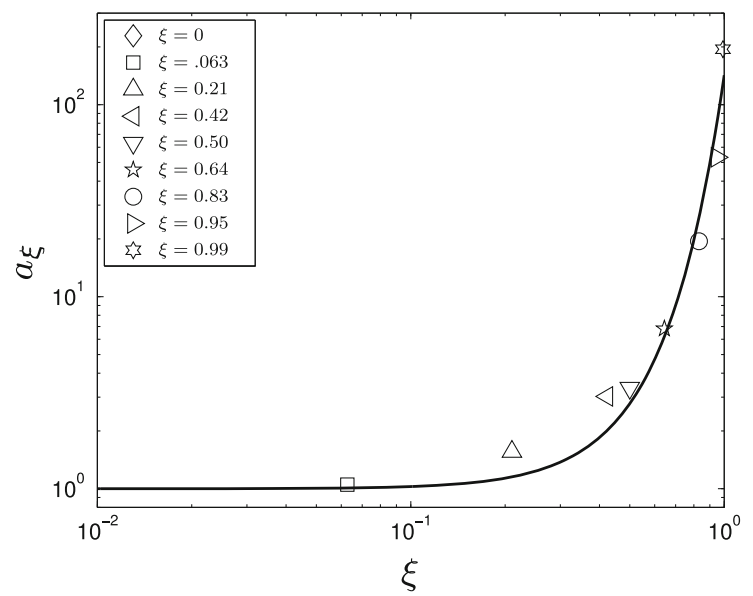

(a) curve constructed in this way (Fig. 3a), with its promising range of eight decades, has no physical meaning. Consequently, this master curve can not be used to obtain the complex viscosity of the pure melt at very high frequency by measuring the complex viscosity of the partly crystallized material at a lower frequency.

For this reason, we propose to use the two-step procedure commonly used in TTS. The result of this procedure is shown in Fig. 5. Contrary to the one-step procedure, this yields a smooth master curve for the phase angle, indicating that the shift factor $a_{\xi}$ obtained in this way is the correct shift factor for the relaxation

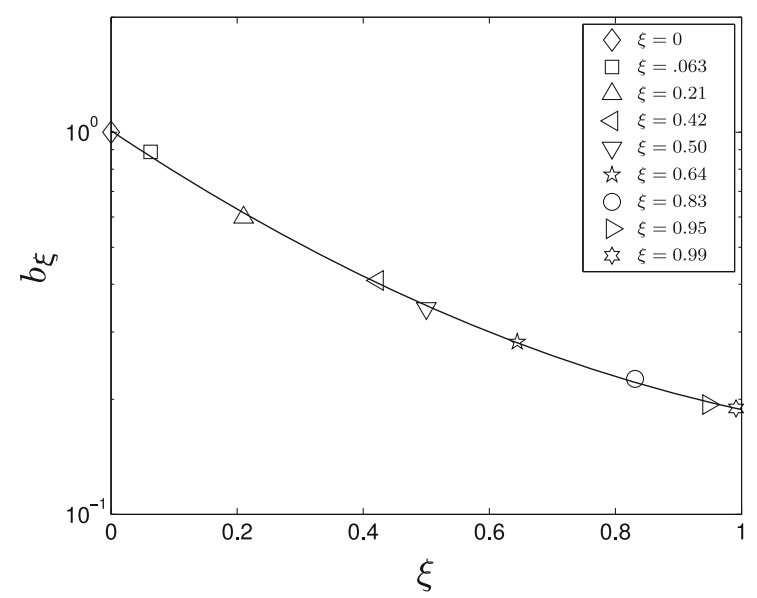

(b)
Fig. 6 Horizontal shift factor $a_{\xi}$, symbols are values to shift the measurements, line is the empirical fit from Lamberti et al. with parameters $\alpha_{1}=2.08, \alpha_{2}=2.56(\mathbf{a})$. Vertical shift factor $b_{\xi}(\xi)$, symbols are values to shift the measurements, line shows a fit of the form $\log _{10}\left(b_{\xi}\right)=\beta_{1} \xi^{2}-\beta_{2} \xi$ with $\beta_{1}=0.22, \beta_{2}=1.01$ (b) 
Table 2 Space filling shift factors

\begin{tabular}{lllll}
\hline$\xi$ & $\begin{array}{l}a_{\xi} \\
(\text { experimental })\end{array}$ & $\begin{array}{l}a_{\xi} \\
\text { (simulations) }\end{array}$ & $\begin{array}{l}b_{\xi} \\
\text { (experiments) }\end{array}$ & $\begin{array}{l}b_{\xi} \\
\text { (simulations) }\end{array}$ \\
\hline 0 & 1.00 & 1.00 & 1.00 & 1.00 \\
0.063 & 1.05 & 1.01 & 0.89 & 0.88 \\
0.21 & 1.56 & 1.19 & 0.60 & 0.65 \\
0.42 & 3.03 & 2.17 & 0.41 & 0.42 \\
0.50 & 3.45 & 2.97 & 0.35 & 0.35 \\
0.64 & 6.81 & 6.35 & 0.28 & 0.28 \\
0.83 & 19.4 & 15.7 & 0.23 & 0.21 \\
0.95 & 53.2 & 32.3 & 0.19 & 0.18 \\
0.99 & 194.4 & 75.3 & 0.19 & 0.17 \\
\hline
\end{tabular}

times of the material. Compare Figs. 4 and 5a. For the complex viscosity we also obtain a smooth master curve. Again the measurements with $\xi=0$ correspond to the values in Table 1. Unfortunately, the frequency range that the master curves span is reduced to four decades. The shift factors that were used to construct these master curves are given in Fig. 6 and Table 2 . Although the procedure for constructing a master curve is different, the empirical fit by Lamberti et al. is still able to describe the data for the timescale shift factor. The values for vertical shift factor $b_{\xi}$ can be described by

$\log _{10} b_{\xi}=\beta_{1} \xi^{2}-\beta_{2} \xi$.

We observe that the phase angle measurements for the lowest frequency $(\omega=1 \mathrm{rad} / \mathrm{s})$ slightly deviate from the master curve, see Fig. 5a. This indicates that time-hardening superposition (THS) might not give a good representation at lower oscillatory frequencies. We come back to this, and to our interpretation of the meaning of the parameters $a_{\xi}$ and $b_{\xi}$, in "Time-hardening superposition with the GSCM" section.

\section{Simulations}

The generalized self-consistent method

The crystallizing melt is modeled as a suspension of spherical soft particles (the spherulites) in a viscoelastic matrix (the amorphous melt). The properties of such a system can be calculated using the three-dimensional (3D) GSCM, which was developed to describe the rheological properties of a suspension of spherical soft particles in an elastic medium. The basic problem of the 3D GSCM is shown in Fig. 7. The suspension is represented by a unit cell consisting of a particle with properties of the dispersed phase and a surrounding matrix shell with properties of the matrix phase. Their radii are $a$ and $b$, respectively. The volume fraction of the particles is given by

$\xi=\left(\frac{a}{b}\right)^{3}$

The unit cell is suspended in an infinitely extending medium, which has the (unknown) effective properties of the suspension. The solution of the three phase problem yields the effective properties of the composite medium of an isotropic matrix phase into which is embedded the isotropic inclusion phase. The result for the effective medium problem of Fig. 7 is given by the solution of the quadratic equation (Christensen and Lo 1979, 1986)

$A f_{G}^{2}+B f_{G}+C=0$

where $f_{G}$ is the relative modulus,

$f_{G}=\frac{G}{G_{0}}$

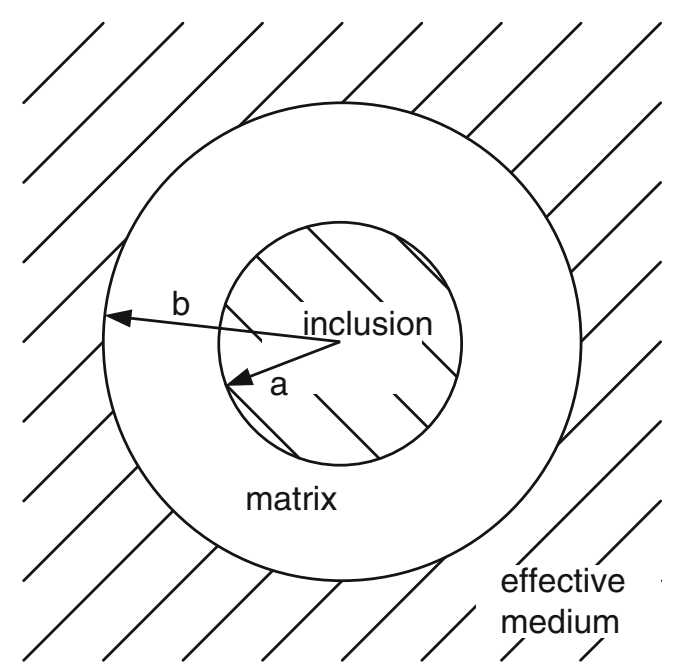

Fig. 7 Generalized self-consistent method, after Fig. 1 in Christensen (1990) 
with $G$ the modulus of the suspension and $G_{0}$ the modulus of the matrix. The coefficients $A, B$ and $C$ depend on space filling $\xi$, the Poisson's ratios of the matrix and dispersed phase, $v_{0}$ and $v_{1}$, respectively, and the ratio between the moduli of the dispersed phase and the matrix, $\mu=G_{1} / G_{0}$. Expressions for the coefficients $A, B$ and $C$ are given in the Appendix. Steenbakkers and Peters (2008) applied the GSCM, derived for elastic materials, to viscoelastic materials by utilizing the correspondence principle Hashin $(1965,1970 \mathrm{a}, \mathrm{b})$. In that case, the relative modulus is complex;

$f_{G}^{*}(\omega)=\frac{G^{*}(\omega)}{G_{0}^{*}(\omega)}$

and so is the modulus ratio $\mu^{*}=G_{1}^{*} / G_{0}^{*}$. The relative modulus can be calculated from

$A^{*} f_{G}^{* 2}+B^{*} f_{G}^{*}+C^{*}=0$

where the complex coefficients $A^{*}, B^{*}$ and $C^{*}$ follow from $A, B$ and $C$ when $\mu$ is replaced by $\mu^{*}$. The absolute value of the complex relative modulus, $\left|f_{G}^{*}\right|$, is equal to the NRF as defined in Eq. 2.

The 3D GSCM was developed for systems with purely spherical dispersed particles. Growing spherulites will impinge at some point and as a result the dispersed particles in this system will not be purely spherical at high volume fractions (above $\sim 70 \%$ ). Previous studies do show a discrepancy between the GSCM and rheology experiments at very high volume fractions $(\sim 98 \%)$

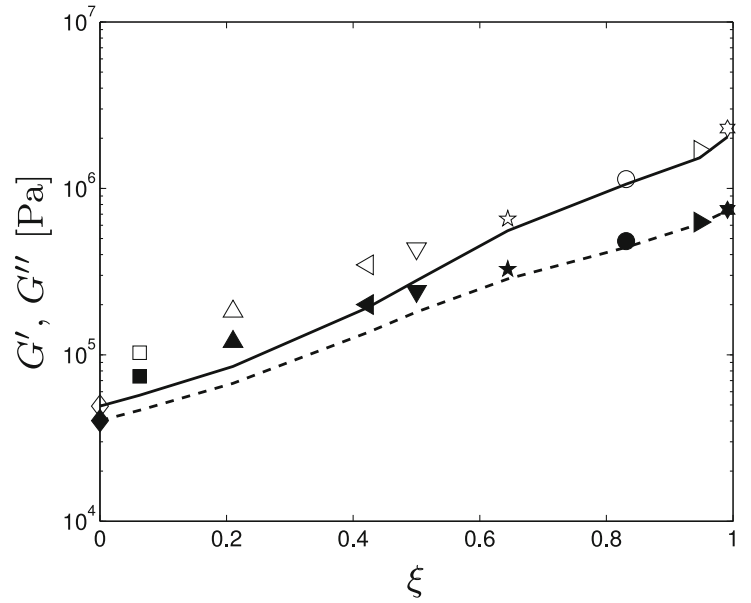

(a)

Fig. 8 Comparison of the storage modulus (open symbols, measurements and solid line, predictions from suspension model) and loss modulus (closed symbols, measurements and dashed line, predictions from suspension model) versus space filling at
(Housmans et al. 2009; Steenbakkers and Peters 2008). However, below this value there seems to be no noticeable effect of impingement on the hardening behavior.

In this study, we examine only quiescent crystallization, with crystalline structures that are spherical (before impingement). Flow-induced crystallization may yield oriented cylindrical crystalline structures. Steenbakkers and Peters (2008) found that these have qualitatively similar hardening behavior but were not able to quantitatively capture the behavior using a $2 \mathrm{D}$ suspension model. More research on this will be done in future work.

\section{Early stage of crystallization}

We observe that the change in material properties from $0 \%$ to $6 \%$ space filling is much larger than would be expected from a suspension. This is illustrated in Fig. 8, where we show measurements and simulations for the storage and loss moduli versus space filling for $\omega=1$ $\mathrm{rad} / \mathrm{s}$. Figure 8 a shows simulations where we have taken the modulus of the pure melt $G_{0}^{*}$ as the measured modulus of the material with no space filling. The simulations consistently underpredict the measured moduli. The discrepancy can be resolved by extrapolating $G_{0}^{*}$ from the data for the crystallizing melt. This was done for the simulations shown in Fig. 8b. With this value for $G_{0}^{*}$ the simulations and experiments show quantitative agreement for the full range of space filling.

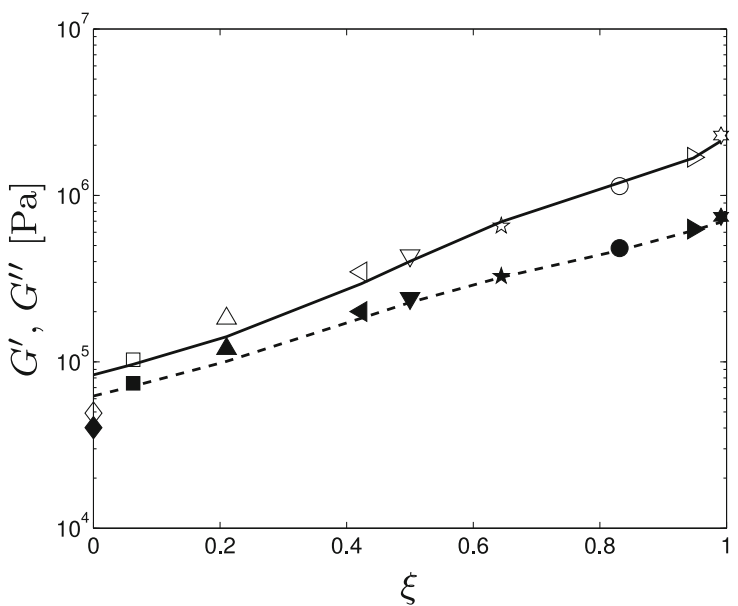

(b)

$\omega=1 \mathrm{rad} / \mathrm{s}$. a shows simulation results with $G_{0}^{*}$ as the measured modulus of the material with no space filling, $\mathbf{b}$ shows simulation results with $G_{0}^{*}$ extrapolated from data for the crystallizing melt (shown in Table 1) 
In our view, this indicates that between $0 \%$ and $6 \%$ space filling a process has taken place that has altered the melt in a way that can not be described by suspension-like behavior. Something very similar is observed in the data from Carrot et al. (1993), who show the evolution of storage and loss modulus for a crystallizing HDPE melt under isothermal conditions at different temperatures. A strong and very steep increase in modulus is seen soon after the onset of crystallization ( $\sim 200$ seconds). After this, the modulus increases gradually until crystallization is complete $\left(10^{3}-10^{4} \mathrm{~s}\right.$, depending on the temperature). Carrot et al. (1993) did not comment on the phenomenon.

Perhaps a network of physical crosslinks is formed (Boutahar et al. 1996, 1998; Coppola et al. 2006; Peters et al. 2002; Pogodina et al. 1999a; Zuidema et al. 2001). Subsequently, spherulites grow in this altered melt, the effect of which is well described by suspension-like

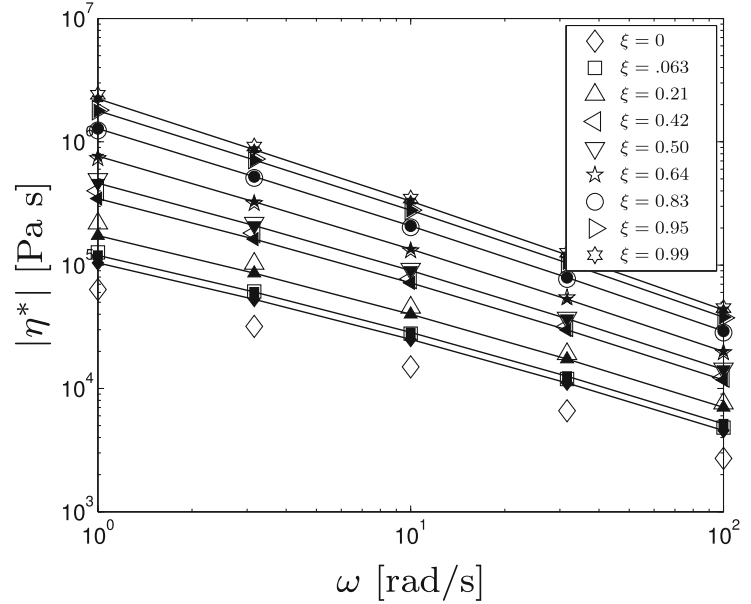

(a)

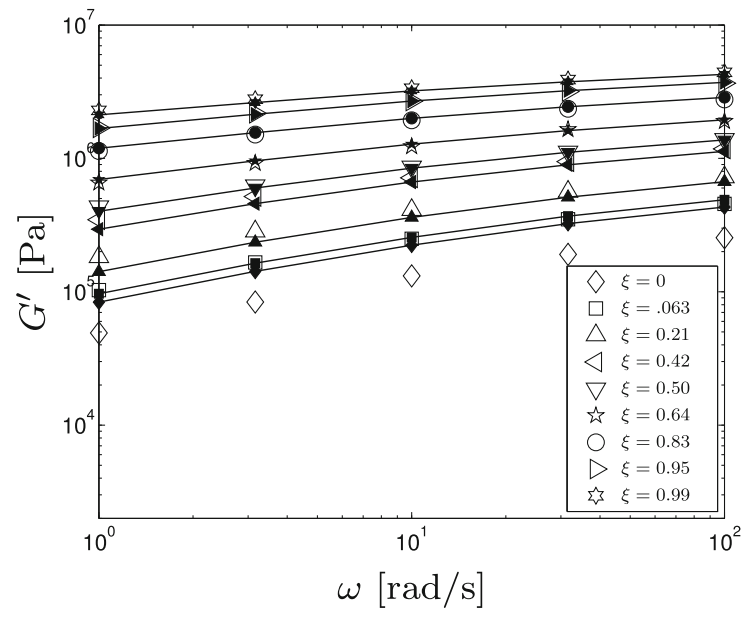

(c) behavior. What exactly happens during the early stages of crystallization remains a lively research topic. For now, we deal with the phenomenon by adjusting the values for the moduli of the melt as described above. Results that are presented in this study are obtained with the adjusted value for the rheology of the melt.

\section{Comparison with experiments}

Figure 9 shows the experimental quantities depicted in Fig. 1 and the results for simulations with the GSCM for the same conditions. Quantitative agreements are observed for the storage and loss modulus and for the magnitude of the complex viscosity (for $\xi \neq 0$, as explained above). For $0.063<\xi<0.64$ the GSCM is less accurate in predicting the phase angle, however agreement is still qualitative.

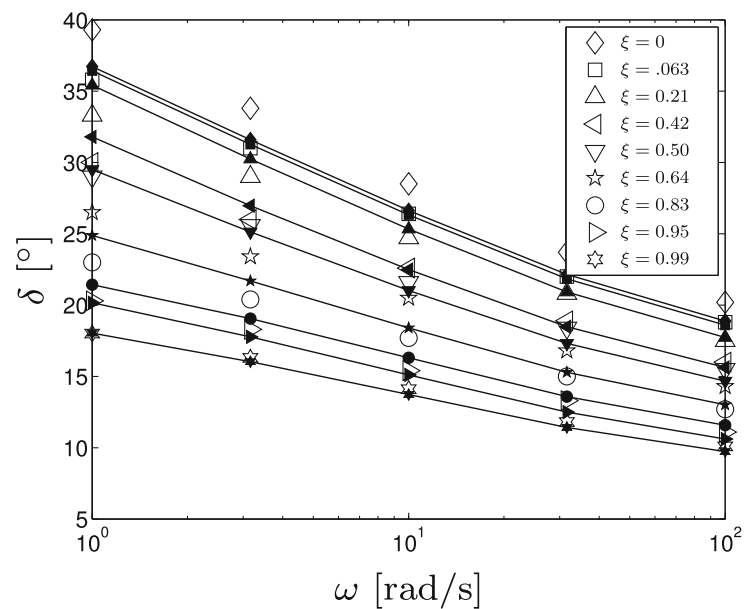

(b)

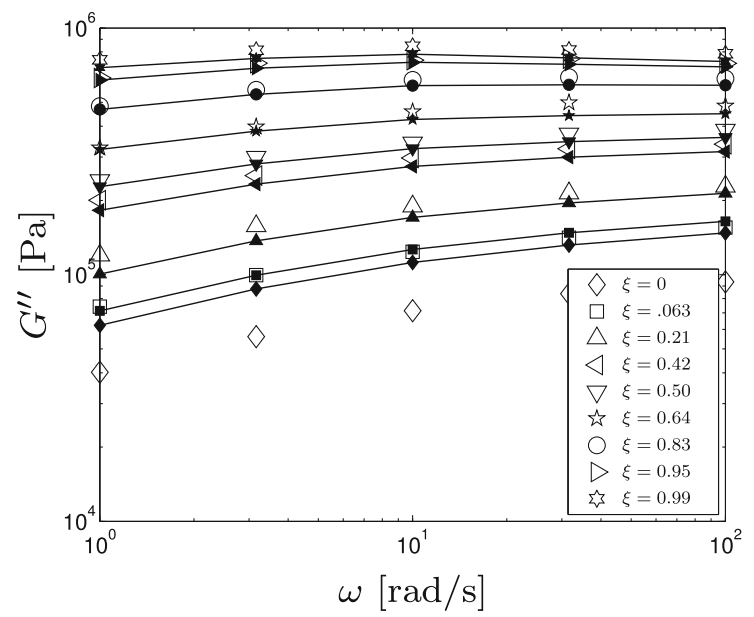

(d)

Fig. 9 Open symbols, measurements of the magnitude of the complex viscosity (a), phase angle (b), storage modulus (c), and loss modulus (d). Closed symbols and solid lines show simulations 


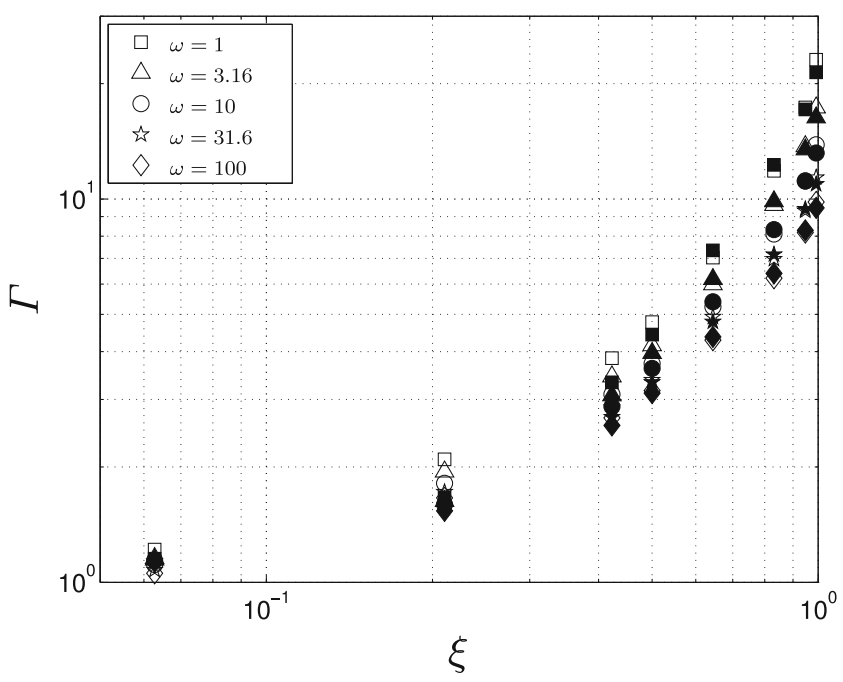

Fig. 10 Hardening curves for five frequencies. Open symbols show measurements and closed symbols show simulations

Hardening function

Figure 10 shows the hardening curve for the material, both from experiments and simulations. We observe quantitative agreement, the hardening function of this material is very well described by suspension-like behavior.

Time-hardening superposition with the GSCM

The master curves for phase angle and viscosity obtained using the two-step procedure are shown in Fig. 11. The corresponding shift factors are given in

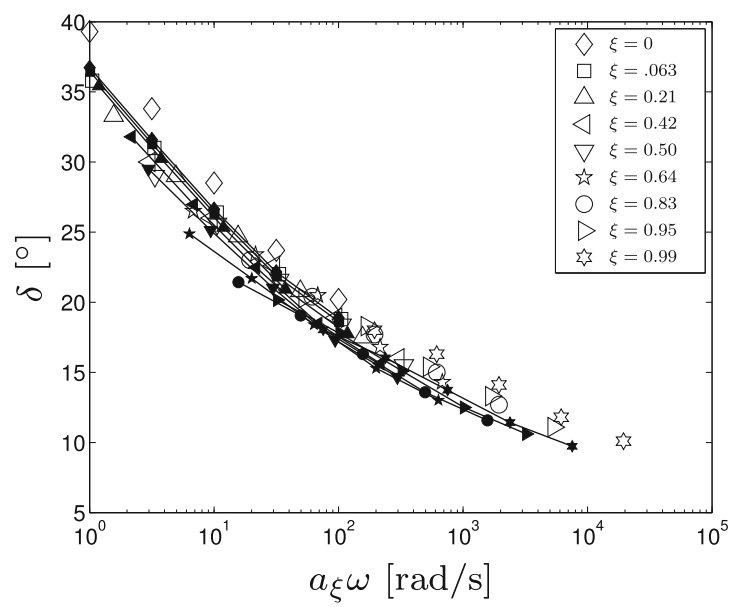

(a)
Fig. 12 and Table 2. We observe a good agreement; the 3D GSCM model can be used to calculate $a_{\xi}$ as well as $b_{\xi}$.

For the lowest frequency measurements and simulations slightly deviate from the phase angle master curve. Because this is observed both for measurements and simulations we cannot attribute this to gel-like behavior, as the simulations capture only suspensionlike behavior. This means that for lower frequencies, THS might be less accurate.

In their review of the applicability of suspension models to polymer crystallization, Steenbakkers and Peters (2008) proposed a phenomenological model for hardening behavior. Suppose a relaxation spectrum $\left(G_{i}, \lambda_{i}\right)$ with $M$ modes is known for the melt so that storage and loss modulus as a function of frequency are given by

$G^{\prime}(\omega)=\sum_{i}^{M} G_{0, i} \frac{\lambda_{0, i}^{2} \omega^{2}}{1+\lambda_{0, i}^{2} \omega^{2}}$,

$G^{\prime \prime}(\omega)=\sum_{i}^{M} G_{0, i} \frac{\lambda_{0, i} \omega}{1+\lambda_{0, i}^{2} \omega^{2}}$.

Now, if the number of modes is the same for both the liquid and solid phase, the moduli and relaxation times of the solid phase can be expressed in terms of the moduli and relaxation times of the matrix as

$G_{i}(\xi)=k_{G, i}(\xi) G_{0, i}$

$\lambda_{i}(\xi)=k_{\lambda, i}(\xi) \lambda_{0, i}$.

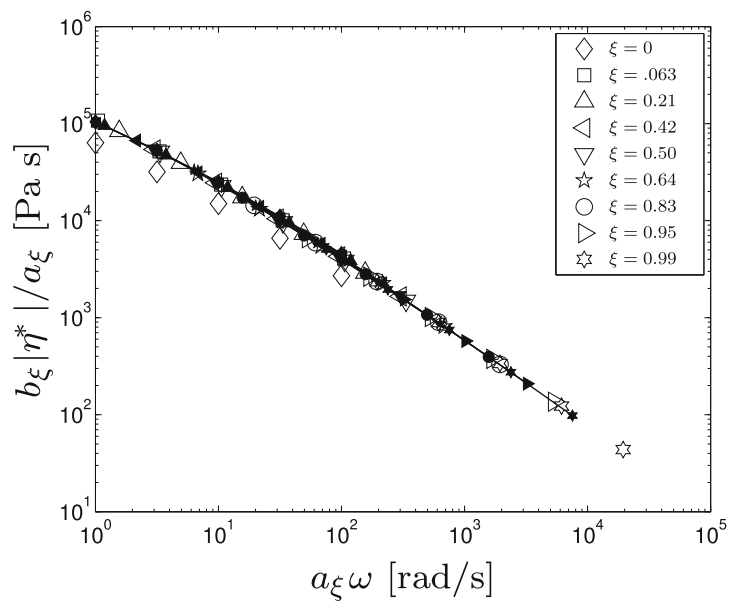

(b)

Fig. 11 Master curves of the phase angle (a) and the viscosity (b). Symbols show measurements and lines show simulations. Data shifted using the two-step procedure 


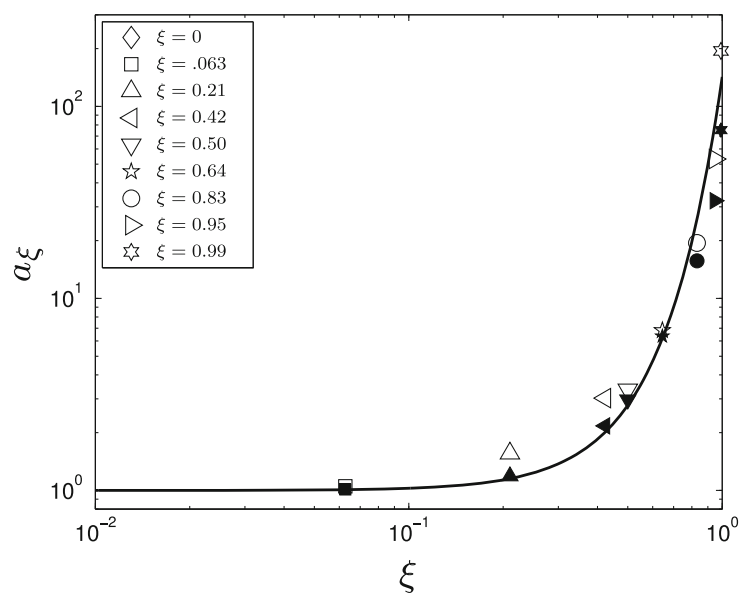

(a)

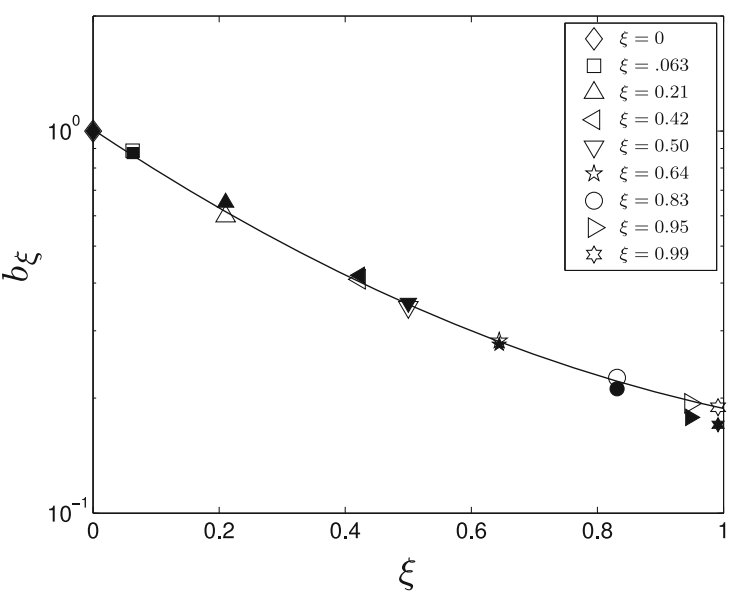

(b)

Fig. 12 Shift factor $a_{\xi}$, open symbols are values to shift the measurements, closed symbols are the shift factors from the master curve from simulations, and line is the empirical fit from Lamberti et al. with parameters $\alpha_{1}=2.08, \alpha_{2}=2.56$ (a). Vertical shift

with $1 \leq k_{G, i} \leq G_{1, i} / G_{0, i}$ and $1 \leq \lambda_{G, i} \leq \lambda_{1, i} / \lambda_{0, i}$. Storage and loss moduli as a function of frequency and spacefilling can then be expressed as

$$
\begin{aligned}
G^{\prime}(\omega, \xi) & =\sum_{i}^{M} k_{G, i}(\xi) G_{0, i} \frac{k_{\lambda, i}(\xi)^{2} \lambda_{0, i}^{2} \omega^{2}}{1+k_{\lambda, i}(\xi)^{2} \lambda_{0, i}^{2} \omega^{2}}, \\
G^{\prime \prime}(\omega, \xi) & =\sum_{i}^{M} k_{G, i}(\xi) G_{0, i} \frac{k_{\lambda, i}(\xi) \lambda_{0, i} \omega}{1+k_{\lambda, i}(\xi)^{2} \lambda_{0, i}^{2} \omega^{2}} .
\end{aligned}
$$

Steenbakkers and Peters did not derive expressions for $k_{G}$ and $k_{\lambda}$. The results obtained here, i.e., the fact that a

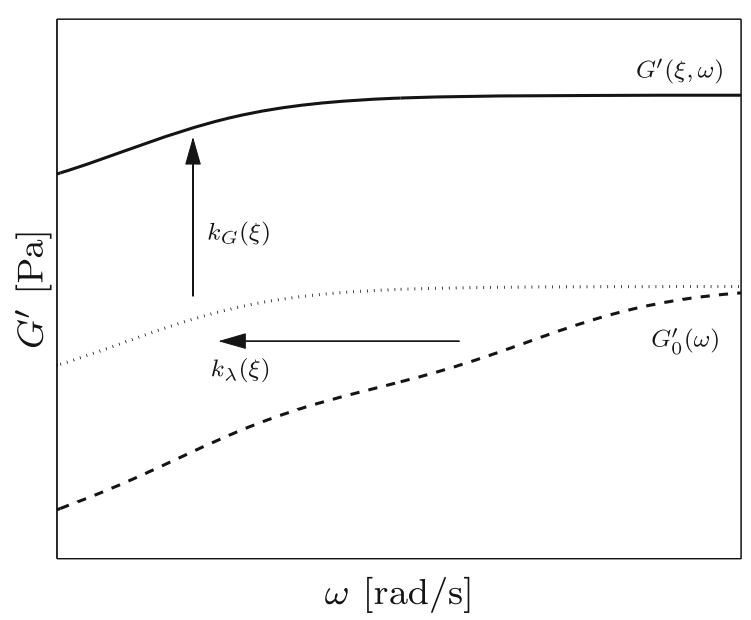

(a)

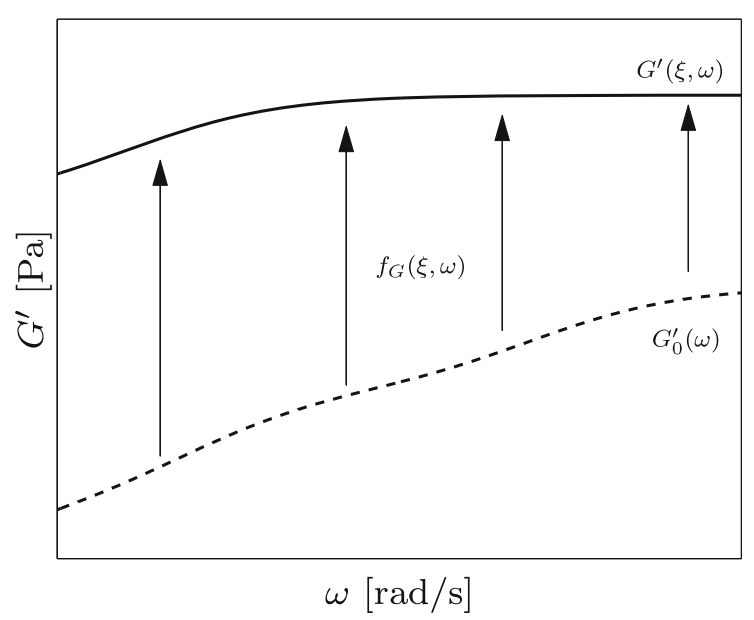

(b)

Fig. 13 Schematic depiction of calculating storage modulus with THS (a) and $f_{G}^{*}(\mathbf{b})$ 
Table 3 Expressions for the coefficients in the 3D GSCM

\begin{tabular}{llll}
\hline $\left.\begin{array}{l}A \\
B \\
C\end{array}\right\}=c_{1}(\mu-1) \eta_{1} \xi^{(10 / 3)}+c_{2}\left[63(\mu-1) \eta_{2}+2 \eta_{1} \eta_{3}\right] \xi^{(7 / 3)}+c_{3}(\mu-1) \eta_{2} \xi^{(5 / 3)}$ & \\
& $+c_{4}(\mu-1) \eta_{2} \xi+c_{5} \eta_{2} \eta_{3}$ & & \\
\hline with... & $\ldots$ for A: & $-4\left(1-5 v_{0}\right)$ & $-4\left(7-5 v_{0}\right)$ \\
\hline$c_{1}$ & $8\left(4-5 v_{0}\right)$ & 4 & -2 \\
$c_{2}$ & -2 & -504 & 252 \\
$c_{3}$ & 252 & $150\left(3-v_{0}\right) v_{0}$ & $-25\left(7-v_{0}^{2}\right)$ \\
$c_{4}$ & $-50\left(7-12 v_{0}+8 v_{0}^{2}\right)$ & $3\left(7-15 v_{0}\right)$ & $-\left(7+5 v_{0}\right)$ \\
$c_{5}$ & $4\left(7-10 v_{0}\right)$ & & \\
$\eta_{1}$ & $(\mu-1)\left(7-10 v_{0}\right)\left(7+5 v_{1}\right)+105\left(v_{1}-v_{0}\right)$ & \\
$\eta_{2}$ & $(\mu-1)\left(7+5 v_{1}\right)+35\left(1-v_{1}\right)$ & & \\
$\eta_{3}$ & 1 & & \\
\hline
\end{tabular}

In this study, we have presented two ways to calculate the rheological properties of a crystallizing material as a function of space filling and oscillatory frequency. Figure 13 schematically shows both, for purpose of clarity. Time-hardening superposition is shown in Fig. 13a; the two shift factors $k_{\lambda}$ and $k_{G}$ are independent of frequency and shift the whole $G^{*}(\omega)$ curve at once. Figure $13 \mathrm{~b}$ shows calculation of the storage modulus via a relative modulus $f_{G}^{*}$ which is calculated for each $\xi$ and $\omega$ using the GSCM.

\section{Conclusions}

The evolution of the rheology of an HDPE during solidification was analyzed. Quantitative agreement with a suspension-based model, the $3 \mathrm{D}$ generalized selfconsistent method, was found. This indicates that in the frequency range investigated, a suspension with spherical soft particles in a viscoelastic matrix is a good representation of an amorphous melt with growing spherulites. In the early stage of crystallization, a phenomenon is observed that can not be explained by suspension-like behavior. This was dealt with by using adjusted values for the complex modulus of the melt.

THS was performed. An improvement on the procedure developed in literature is proposed. The fact that a THS master curve can be constructed means that with a known Maxwell spectrum of the melt, the properties of a crystallizing melt can be obtained using two shift parameters that are mode and frequency independent and which can be easily measured or obtained from a suspension-based model.

Acknowledgments This work is part of a collaboration with the group of Prof. Julia Kornfield at the California Institute of Technology. The project is supported by the Dutch Technology Foundation (STW), grant no. 08083 and the National Science Foundation, grant no. DMR-0710662.
Open Access This article is distributed under the terms of the Creative Commons Attribution Noncommercial License which permits any noncommercial use, distribution, and reproduction in any medium, provided the original author(s) and source are credited.

\section{Appendix}

\section{Coefficients in the GSCM}

Expressions for the coefficients $A, B$, and $C$ in the 3D generalized self-consistent model derived by Christensen (1990), Christensen and Lo $(1979,1986)$ are shown in Table 3.

\section{Poisson's ratios}

To solve Eq. 12, the Poisson's ratios of the continuous and dispersed phase are required. They can be calculated using the well-known formula (Timoshenko and Goodier 1951)

$v=\frac{3 K-2 G}{2(3 K+G)}$,

where $K$ is the bulk modulus and $G$ is the shear modulus. The bulk modulus of HDPE $K \approx 1.5 \mathrm{GPa}$ (Aleman 1990). With the plateau for the storage modulus at approximately $0.4 \mathrm{MPa}$ for $\xi=0$ and at approximately $6 \mathrm{MPa}$ for $\xi \approx 1$ (see Fig. 1 ), we find estimates of $v_{0}=$ $0.4999, v_{1}=0.4980$.

\section{References}

Acierno S, Grizzuti N (2003) Measurements of the rheological behavior of a crystallizing polymer by an "inverse quenching" technique. J Rheol 47(2):563-576 
Alberola N, Mele P (1996) Viscoelasticity of polymers filled by rigid or soft particles: theory and experiment. Polym Compos 17(5):751-759

Aleman J (1990) Bulk and surface compression viscositeis of high and low density polyethylenes. Polym Eng Sci 30(6):326334

Ball R, Richmond P (1980) Dynamics of colloidal dispersions. Phys Chem Liq 9(2):99-116

Batchelor G (1977) Effect of brownian motion on bulk stress in a suspension of spherical particles. J Fluid Mech 83:97117

Boutahar K, Carrot C, Guillet J (1996) Polypropylene during crystallization from the melt as a model for the rheology of molten-filled polymers. J Appl Polym Sci 60:103-114

Boutahar K, Carrot C, Guillet J (1998) Crystallization of polyolefins from rheological measurements-relation between the transformed fraction and the dynamic moduli. Macromolecules 31:1921-1929

Carrot C, Guillet J, Boutahar K (1993) Rheological behavior of a semi-crystalline polymer during isothermal crystallization. Rheol Acta 32:566-574

Christensen R (1990) A critical evaluation for a class of micromechanics models. J Mech Phys Solids 38:379-404

Christensen R, Lo K (1979) Solutions for effective shear properties in three phase sphere and cylinder models. J Mech Phys Solids 27:315-330

Christensen R, Lo K (1986) Erratum: solutions for effective shear properties in three phase sphere and cylinder models. J Mech Phys Solids 34:639

Coppola S, Acierno S, Grizzuti N, Vlassopoulos D (2006) Viscoelastic behavior of semicrystalline thermoplastic polymers during the early stages of crystallization. Macromolecules 39:1507-1514

Custódio F, Steenbakkers R, Anderson P, Peters G, Meijer H (2009) Model development and validation of crystallization behavior in injection molding prototype flows. Macromol Theory Simul 18(9):469-494

Doufas A, McHugh A, Miller C (2000) Simulation of melt spinning including flow-induced crystallization - part I. Model development and predictions. J Non-Newton Fluid Mech 92(1):27-66

Einstein A (1906) A new determination of molecular dimensions. Ann Phys 19:289-306

Einstein A (1911) Correction to: a new determination of molecular dimensions. Ann Phys 34:591-592

Frankel N, Acrivos A (1967) On viscosity of a concentrated suspension of solid spheres. Chem Eng Sci 22(6):847-853

Graham A (1981) On the viscosity of suspensions of solid spheres. Appl Sci Res 37(3-4):275-286

Han S, Wang K (1997) Shrinkage prediction for slowlycrystallizing thermoplastic polymers in injection molding. Int Polym Process 12(3):228-237

Hashin Z (1965) Viscoelastic behavior of heterogeneous media. J Appl Mech 32(3):630-636

Hashin Z (1970a) Complex moduli of viscoelastic compositesI. General theory and application to particulate composites. Int J Solids Struct 6:539-552

Hashin Z (1970b) Complex moduli of viscoelastic compositesII. Fiber reinforced materials. Int J Solids Struct 6:797807

Hieber C (2002) Modeling/simulating the injection molding of isotactic polypropylene. Polym Eng Sci 42(7):13871409

Housmans JW, Steenbakkers RJA, Roozemond PC, Peters GWM, Meijer HEH (2009) Saturation of pointlike nuclei and the transition to oriented structures in flow-induced crystallization of isotactic polypropylene. Macromolecules 42:5728-5740

Janssens V, Block C, van Assche G, van Mele B, van Puyvelde P (2009) RheoDSC: design and validation of a new hybrid measurement technique. J Therm Anal Calorim 98(3):675681

Janssens V, Block C, van Assche G, van Mele B, van Puyvelde P (2010) RheoDSC: analysis of the hardening of semicrystalline polymers during quiescent isothermal crystallization. Int Polym Process 25(4):304-310

Katayama K, Yoon M (1985) Chapter 8. Polymer crystallization in melt spinning: mathematical simulation. In: Ziabicki A, Kawai H (eds) High speed fibre spinning. Wiley, New York

Katoaka T, Kitano T, Sasahara M, Nishijima K (1978) Viscosity of particle filled polymer melts. Rheol Acta 17(2):149 155

Khanna Y (1993) Rheological mechanism and overview of nucleated crystallization kinetics. Macromolecules 26(14):3639-3643

Kiewiet S, Janssens V, Miltner H, Assche G, van Puyvelde P, van Mele B (2008) RheoDSC: a hyphenated technique for the simultaneous measurement of calorimetric and rheological evolutions. Rev Sci Instrum 79(2 Pt 1):023905

Kitano T, Katoaka T, Shirota T (1981) An empirical equation of the relative viscosity of polymer melts filled with various inorganic fillers. Rheol Acta 20(2):207-209

Krieger I, Dougherty T (1959) A mechanism for non-newtonian flow in suspensions of rigid spheres. Trans Soc Rheol 3:137152

Lamberti G, Peters GWM, Titomanlio G (2007) Crystallinity and linear rheological properties of polymers. Int Polym Process 22(3):303-310

Mooney M (1951) The viscosity of a concentrated suspension of spherical particles. J Colloid Sci 6(2):162-170

Pantani R, Speranza V, Titomanlio G (2001) Relevance of moldinduced thermal boundary conditions and cavity deformation in the simulation of injection molding. Polym Eng Sci 41(11):2022-2035

Peters G, Swartjes F, Meijer H (2002) A recoverable strainbased model for flow-induced crystallization. Macromol Symp 185:277-292

Pogodina N, Winter H (1998) Polypropylene crystallization as a physical gelation process. Macromolecules 31(23):8164-8172

Pogodina N, Siddiquee S, Egmond J, Winter H (1999a) Correlation of rheology and light scattering in isotactic polypropylene during early stages of crystallization. Macromolecules 32:1167-1174

Pogodina N, Winter H, Srinivas S (1999b) Strain effects on physcial gelation of crystallizing isotactic polypropylene. J Polym Sci, B, Polym Phys 37:3512-3519

Shimizu J, Okui N, Kikutani T (1985) Chapter 15. Fine structure and physical properties of fibers melt-spun at high speeds from various polymers. In: Ziabicki A, Kawai H (eds) High speed fibre spinning. Wiley, New York

Steenbakkers R, Peters G (2008) Suspension-based rheological modeling of crystallizing polymer melts. Rheol Acta 47(56):643-665

Steenbakkers R, Peters G (2010) A stretch-based model for flowenhanced nucleation of polymer melts. J Rheol 55(2):401-433

Swartjes F, Peters G, Rastogi S (2003) Stress induced crystallization in elongational flow. Int Polym Process 18:53-66

Tanner R (2003) On the flow of crystallizing polymers. I. Linear regime. J Non-Newton Fluid Mech 112(2-3):251-268

Timoshenko S, Goodier J (1951) Theory of elasticity. McGrawHill Book Company, Inc

Titomanlio G, Speranza V, Brucato V (1997) On the simulation of thermoplastic injection moulding process. 2. Relevance 
of interaction between flow and crystallization. Int Polym Process 12(1):45-53

Winter H, Mours M (1997) Rheology of polymers near liquidsolid transitions. In: Neutron spin echo spectroscopy, viscoelasticity, rheology, vol 134, pp 165-234

Yarin A (1992) Flow-induced online crystallization of rodlike molecules in fibre spinning. J Appl Polym Sci 46(5):873-878
Ziabicki A (1988) The mechanisms of neck-like deformation in high-speed melt spinning. 1. Rheological and dynamic factors. J Non-Newton Fluid Mech 30(2-3):141-155

Zuidema H, Peters G, Meijer H (2001) Development and validation of a recoverable strain-based model for flow-induced crystallization of polymers. Macromol Theory Simul 10:447460 